\title{
X-Ray Attenuation Cell
}

\author{
D. Ryutov, A. Toor
}

March 3, 2000

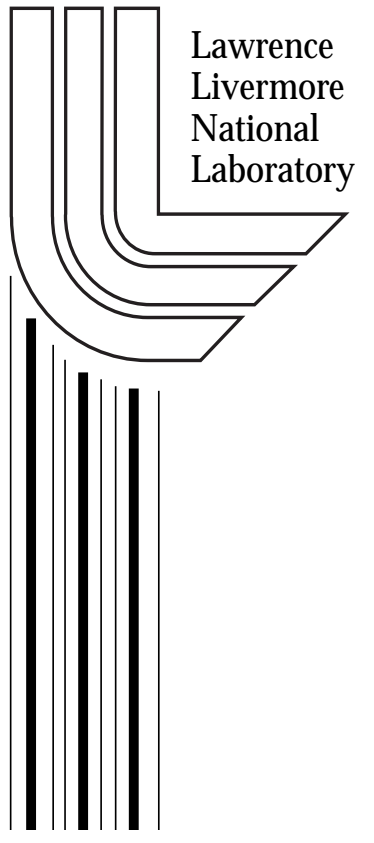




\section{DISCLAIMER}

This document was prepared as an account of work sponsored by an agency of the United States Government. Neither the United States Government nor the University of California nor any of their employees, makes any warranty, express or implied, or assumes any legal liability or responsibility for the accuracy, completeness, or usefulness of any information, apparatus, product, or process disclosed, or represents that its use would not infringe privately owned rights. Reference herein to any specific commercial product, process, or service by trade name, trademark, manufacturer, or otherwise, does not necessarily constitute or imply its endorsement, recommendation, or favoring by the United States Government or the University of California. The views and opinions of authors expressed herein do not necessarily state or reflect those of the United States Government or the University of California, and shall not be used for advertising or product endorsement purposes.

This work was performed under the auspices of the U. S. Department of Energy by the University of California, Lawrence Livermore National Laboratory under Contract No. W-7405-Eng-48.

This report has been reproduced directly from the best available copy.

Available to DOE and DOE contractors from the

Office of Scientific and Technical Information

P.O. Box 62, Oak Ridge, TN 37831

Prices available from (423) 576-8401

http://apollo.osti.gov/bridge/

Available to the public from the National Technical Information Service

U.S. Department of Commerce 5285 Port Royal Rd., Springfield, VA 22161

http://www.ntis.gov/

OR

Lawrence Livermore National Laboratory Technical Information Department's Digital Library http://www.llnl.gov/tid/Library.html 


\title{
X-ray attenuation cell
}

\author{
D. Ryutov, A. Toor \\ Lawrence Livermore National Laboratory
}

\section{Introduction}

To minimize the pulse-to-pulse variation, the LCLS FEL must operate at saturation, i.e. 10 orders of magnitude brighter spectral brilliance than $3^{\text {rd }}$-generation light sources. At this intensity, ultra-high vacuums and windowless transport are required. Many of the experiments, however, will need to be conducted at a much lower intensity thereby requiring a reliable means to reduce the $\mathrm{x}$-ray intensity by many orders of magnitude without increasing the pulse-to-pulse variation. In this report we consider a possible solution for controlled attenuation of the LCLS x-ray radiation. We suggest using for this purpose a windowless gas-filled cell with the differential pumping. Although this scheme is easily realizable in principle, it has to be demonstrated that the attenuator can be made short enough to be practical and that the gas loads delivered to the vacuum line of sight (LOS) are acceptable. We are not going to present a final, optimized design. Instead, we will provide a preliminary analysis showing that the whole concept is robust and is worth further study.

The spatial structure of the LCLS x-ray pulse at the location of the attenuator is shown in Fig. 1. The central high-intensity component, due to the FEL, has a FWHM of $\sim 100 \mu \mathrm{m}$. A second component, due to the undulator's broad band spontaneous radiation is seen as a much lower intensity "halo" with a FWHM of $1 \mathrm{~mm}$. We discuss two versions of the attenuation cell. The first is directed towards a controlled attenuation of the FEL up to the 4 orders of magnitude in the intensity, with the spontaneous radiation halo being eliminated by collimators. In the second version, the spontaneous radiation is not sacrificed but the FEL component (as well as the first harmonic of the spontaneous radiation) gets attenuated by a more modest factor up to 100 .

We will make all the estimates assuming that the gas used in the attenuator is Xenon and that the energy of the FEL is $8.25 \mathrm{keV}$. At lower FEL energies the problems associated with the attenuator become much easier and reformulation of our results for the case of other gases is straightforward. 
Some important parameters of Xe are as follows. The diameter of the Xe atom is $\mathrm{d} \sim 2.5$ $\AA$ [1, p. 302]. Accordingly, we estimate Xe-Xe collision cross-section as

$$
\sigma \approx \pi d^{2} \approx 2 \cdot 10^{-15} \mathrm{~cm}^{-2} .
$$

At normal conditions (atmospheric pressure, room temperature) the mean-free path for $\mathrm{Xe}$ atoms is

$$
\ell_{\text {atm }} \approx 2 \cdot 10^{-5} \mathrm{~cm} ;
$$

at other densities it is

$$
\ell=\ell_{\text {atm }} \frac{n_{\text {atm }}}{n},
$$

where $n_{a t m}$ is a number density at normal conditions,

$$
n_{\text {atm }} \approx 2.7 \cdot 10^{19} \mathrm{~cm}^{-3} .
$$

The sound speed in Xe at normal conditions is

$$
s_{0} \approx 2 \cdot 10^{4} \mathrm{~cm} / \mathrm{s} .
$$

Kinematic viscosity of the gas is

$$
v \sim \frac{s \ell}{3} .
$$

For Xe at normal conditions it is $\sim 0.1 \mathrm{~cm}^{2} / \mathrm{s}$.

The absorption coefficient at $8.25 \mathrm{keV}$ is taken from Ref. [2]

$$
\mu\left(\mathrm{cm}^{-1}\right) \approx 1.7 \frac{p}{p_{\text {atm }}},
$$

where we assume that the gas is at room temperature. Attenuation $A$ is

$$
A=\exp (\mu x)
$$


Note, that we introduce the attenuation in such a way that it is greater than 1. Attenuation of, say, 100 means that the energy flux is $1 / 100$ of its initial value. According to (6), to reach the attenuation $A=10^{4}$, one has to have a path length $x$ in xenon at the atmospheric pressure

$$
x=5.4 \mathrm{~cm}
$$

At a 5-times smaller pressure, the same attenuation would occur at a path length of 27 $\mathrm{cm}$; attenuation by a factor of 100 would occur at a path length of $13.5 \mathrm{~cm}$.

\section{Attenuator for the central part of the beam}

A schematic representation of this attenuator is shown in Fig. 2. The X-ray beam comes from the right and exits the cell to the left. The gas is fed into the central volume 1 through the gas inlet 2 at the top and then flows out of the central volume through the nozzles 3 and 4. Even a short cell provides, according to Eq. (6), a sufficient and easily controllable (by the gas pressure) attenuation. A key element of this design is the nozzle 3 directed towards the X-ray source. We want to make its entrance hole as small as possible in order to maintain an acceptable gas load on the LCSC vacuum LOS. We will design the nozzle in such a way that the gas jet at its exit has a divergence of $1 / 4$ radian and then deflect the flow from the line of sight, thereby reducing the gas load on the differential pumping system.

We will make all the estimates for the gas pressure in the central volume (1 in Fig.2) equal to 0.2 of the atmospheric pressure

$$
n_{0}=5.4 \cdot 10^{18} \mathrm{~cm}^{-3}
$$

Let us consider a numerical example: the radius $r_{0}$ of the entrance hole ( 5 in Fig. 2 ) equal to

$$
r_{0}=0.5 \mathrm{~mm}
$$

and the radius of the exit hole of the nozzle (6 in Fig. 2)

$$
r_{1}=5 \mathrm{~mm}
$$


The gas density decreases, roughly speaking, by a factor of 100 from the entrance to the exit hole.

According to the estimates (2) and (3), the flow is strongly collisional (hydrodynamical) over the whole length of the nozzle. By making the walls of the nozzle smooth, one can guarantee that the flow will be laminar. Indeed, the Reynolds number, according to Eq. (5) is

$$
\operatorname{Re} \sim \frac{\mathrm{v} r}{v} \sim \frac{3 r}{\ell}
$$

and does not exceed $10^{3}$; in other words, it is below the critical Reynolds number for the onset of the instability in the smooth nozzle.

A 100-fold density reduction leads to an adiabatic cooling of the monatomic gas to the temperature which is $(100)^{2 / 3} \sim 20$ times less than the initial temperature. With the parallel velocity corresponding to approximately a sound speed $s_{0}$, this means that, for a nozzle with a gently increasing radius, one can obtain a weakly divergent jet, with the divergence angle $\theta \sim 1 / \sqrt{20} \sim 0.25 \mathrm{rad}$. We will rotate the direction of the nozzle by a somewhat larger angle away from the axis (Fig. 2). With this, the edge of the nozzle should not block the 1-mm diameter entrance hole. Such arrangements can be made with a nozzle that is $5-6 \mathrm{~cm}$ long.

The main jet is diverted away from the line of sight to the vacuum port(s) by the deflection screen 7 . The density on the axis of the nozzle near the exit orifice (point $\mathrm{M}$ in Fig. 2) is $\sim 100$ times less than the initial density, i.e., it is $\sim 3 \cdot 10^{16} \mathrm{~cm}^{-3}$. A conservative estimate for the density in the "wing" of the jet, near or beyond the $0.25 \mathrm{rad}$ angle (point $\mathrm{N}$ in Fig. 2) is an order of magnitude less, i.e.,

$$
n_{1} \sim 3 \cdot 10^{15} \mathrm{~cm}^{-3}
$$

We will return shortly to the problem of the further density reduction, while now we will estimate the amount of gas that has to be pumped out from the chamber 8. According to Ref. 3, the number of atoms leaving the cell 1 through the nozzle 5 per unit time is

$$
Q_{0}=\pi r_{0}^{2} s_{0} n_{0}\left(\frac{2}{\gamma+1}\right)^{\frac{\gamma+1}{2(\gamma-1)}}
$$


For the parameters of the nozzle given above, the density $n_{0}$ in the attenuating cell 1 defined by Eq. (9), and $\gamma=5 / 3$, one has

$$
Q_{0} \approx 5 \cdot 10^{20} \text { atoms } / \mathrm{s}
$$

To maintain the average density in the cell 8 at the level of $\sim 2 \cdot 10^{15} \mathrm{~cm}^{-3}$, one has to have a pump speed $\sim 2001 / \mathrm{s}$, which can be easily reached with various types of pumps, if the surface area of the vacuum ports in the side walls of the volume 6 is greater than $\sim 100$ $\mathrm{cm}^{2}$.

The gas pumped out of the cell 8 would be returned to the cell 1 , so that there will be essentially no gas loss from this cell. In principle, the gas pumped out from the subsequent sections could also be recovered, especially if cryopumps are used in these sections. On the other hand, the amount of gas leaking beyond the cell 8 , is very small, and probably not worth recovering.

Return now to the problem of a further reduction of the gas density along the line of sight. We suggest to use a 1-mm diameter diaphragm 9 aligned with the entrance orifice 3. The gas stream hits the conical surface at the entrance of this diaphragm and acquires a significant ( 45 degrees) divergence. So, when entering the final stage (10, Fig. 2$)$ of the differential pumping system, it has a significant degree of divergence. The total number of atoms entering the cell 10 is

$$
Q_{1} \approx \pi r_{0}^{2} n_{1} s_{0} \approx 5 \cdot 10^{17} \text { atoms } / s,
$$

where $n_{1}$ is as in Eq. (13). Let the length $L_{2}$ of this last stage be $30 \mathrm{~cm}$. Then, for a 45 degree divergence, the imprint of the atomic beam at the opposite side of the second cell will have a surface area $\pi L^{2}$, and the atomic flux from the attenuator to the high vacuum line of sight will be

$$
Q_{2} \approx \frac{r_{0}^{2}}{L_{2}^{2}} Q_{1} \approx 1.4 \cdot 10^{12} \text { atoms } / \mathrm{s}
$$

The particle density in the atomic stream is

$$
n_{2} \sim 8 \cdot 10^{9} \mathrm{~cm}^{-3} \text {. }
$$


The number of atoms streaming into the accelerator vacuum LOS through the hole 11 for several pressures in the main attenuation cell is shown in Table 1.

\begin{tabular}{|l|l|l|}
\hline Pressure (Torr) & Attenuation & Gas Outflux Q (atoms/s) \\
\hline 152 & $10^{4}$ & $1.4 \times 10^{12}$ \\
\hline 76 & $10^{2}$ & $7.0 \times 10^{11}$ \\
\hline 38 & $10^{1}$ & $3.5 \times 10^{11}$ \\
\hline 19 & 3 & $1.7 \times 10^{11}$ \\
\hline
\end{tabular}

Table 1 .

There will also be particles that experience reflections from the walls of the cell and whose distribution function is almost isotropic. To have their density at the level of $n_{2}$ or below, one would have to provide a high pump speed, $\sim \pi L_{2}^{2} s_{0} \sim 6 \cdot 10^{7} \mathrm{~cm}^{3} / \mathrm{s} \sim 6 \cdot 10^{4} \mathrm{l} / \mathrm{s}$. This can be obtained by using sufficiently large ports in the side walls of the cell 10, opening to a volume with cryopanels. The surface area of these ports should be $>0.3 \mathrm{~m}^{2}$, and the ducts must be short. There is enough room to accommodate these ports on the side walls of the cell 10. One can also reduce the pump speed requirements by adding a simple additional element to the hole 11 (Fig. 2), namely, adding a 1-cm long tube with internal baffles (12, Fig. 2). This would reduce the flow rate of the isotropic component by an order of magnitude, and lead to an order-ofmagnitude reduction of the pump speed. The total length of the whole attenuation cell will be $x+2 L_{1}+2 L_{2} \approx 110 \mathrm{~cm}$ (see Fig. 2 for the notation).

One could also add baffles closer to the hole 9 (Fig. 2) and use intermediate pump systems, but this is probably not needed, because the results with even this very simple system look satisfactory. 


\section{A wide-aperture attenuator.}

In this case the attenuator will have 1-cm diameter apertures. In order to reduce the gas loading on the ultra high vacuum LOS, we reduce the gas density in the cell 1 (Fig 3) by a factor of 10 compared to the previous case, i.e., to

$$
n_{o}=5 \cdot 4 \cdot 10^{17} \mathrm{~cm}^{-3} .
$$

To provide a 100-fold attenuation of 8.25-kev X-rays, the length of the cell 1, according to Eqs. (6) and (7) has to be approximately $135 \mathrm{~cm}$. The mean free path at the density (18) is, according to (3), $\sim 10^{-3} \mathrm{~cm}$. So, again, we have initially a hydrodynamic flow. However, because of a very large aperture, we will handle it in a different way than in the previous design. We will use a straight tube 3 with baffles in it, with the distance between the baffles of the order of the tube radius (Fig. 3). This will make the flow turbulent and provide some increased resistance to the gas flow. The length of the tube will be $\sim 5 \mathrm{~cm}$. (Even if the tube length were increased by a factor of 5-10, the gas outflow will not be greatly reduced because we are still in the hydrodynamic, lowviscosity regime. Since we want to keep the overall length of the attenuator as short as possible, assessment of the overall benefit in increasing the length of the tube beyond 5 $\mathrm{cm}$ will require optimization of the whole design). One can expect that the flow velocity at the entrance hole will then be reduced compared to the sound speed by roughly a factor of 2. So, the total amount of gas escaping the through the hole 2 will be

$$
Q_{0} \approx \pi r_{0}^{2} n_{0} \frac{s_{0}}{2} \approx 4.2 \cdot 10^{21} \text { atoms } / s .
$$

Unlike the previous case, the temperature of the gas on the other side of the tube 3 will be approximately the same as the initial temperature and the divergence angle at the exit from the tube will be approximately 45 degrees. Accordingly, at the distance $L_{1} \sim 10 \mathrm{~cm}$ from the end of the tube 3 (point M in Fig. 3), we will have a particle density

$$
n_{1} \approx \frac{Q_{0}}{\pi L_{1}^{2} s_{0}} \approx n_{0} \frac{r_{0}^{2}}{2 L_{1}^{2}} \approx 7 \cdot 10^{14} \mathrm{~cm}^{-3} .
$$

Maintaining an average vacuum in the cell 4 corresponding to this average density will require a pump speed that can be determined by dividing $Q_{0}$ by $n_{l}$, and is equal to $6 \cdot 10^{3}$ 1/s. The surface area available on the sides of the intermediate volume is more than sufficient to provide this pump speed. If the ports open into large tanks situated around 
the absorption cells, one reaches further reduction of the gas pressure there, and enters the domain where turbopumps can be efficiently used [4].

At a distance of $10 \mathrm{~cm}$ from the end of the tube 3, we will install a nozzle of a special shape (5 in Fig. 3). The gas flow at this stage is still collisional, with the collision meanfree path comparable to the hole size (see Eqs. (2) and (3)). This nozzle creates conditions where the cold gas entering the nozzle at a sound speed experiences reflections from the walls and returns to the room temperature; accordingly, after leaving the nozzle, it diverges at an angle $\sim 45$ degrees into the cell 6 . The total amount of gas entering the cell 6 (Fig. 3) is

$$
Q_{1}=\pi r_{0}^{2} n_{1} s_{0} \sim 10^{19} \text { atoms } / s
$$

Behind the nozzle 5, we already have a molecular flow diverging at an angle $\sim 45^{0}$. At a distance $L_{2} \sim 10 \mathrm{~cm}$ the density drops to

$$
n_{2} \sim \frac{Q_{1}}{\pi L_{2}^{2} s_{0}} \sim 2 \cdot 10^{12} \mathrm{~cm}^{-3}
$$

Adding a few-centimeter long tube allows one to have a relatively high density of the isotropic component in the cell $6, \sim 2 \cdot 10^{13} \mathrm{~cm}^{-3}$, at which the required pump speed for the cell 6 becomes modest, $\sim 6 \cdot 10^{3} 1 /$ s. The total amount of gas leaking into the cell 8 (Fig.3) is

$$
Q_{2} \sim \pi r_{0}^{2} n_{2} s_{0} \sim 3 \cdot 10^{16} \text { atoms } / s .
$$

The mean-free path in the tube is $\sim 10 \mathrm{~cm}$, comparable to the length of the tube. Under such circumstances, the angular divergence of the atomic stream entering the chamber 8 , will be $\theta \sim r_{d} / L_{2} \sim 1 / 20$. At the length of $L_{3} \sim 1 \mathrm{~m}$ downstream, the radius of the atomic flow will become $\theta L_{3} \sim 5 \mathrm{~cm}$, so that the amount of gas leaving the gas cell through the final hole will be

$$
Q_{3} \sim Q_{2} \frac{r_{0}^{2}}{\theta^{2} L_{3}^{2}} \sim Q_{2} \frac{L_{2}^{2}}{L_{3}^{2}} \sim 3 \cdot 10^{14} \text { atoms } / \mathrm{s}
$$

The total length of this system would be approximately $4 \mathrm{~m}$, with the main contribution coming from the outermost cells. 
If the gas influx (24) is still too high for the nominal beam-line vacuum system, one could use a more sophisticated connection between the sections, with a much higher vacuum resistance. This would be a honeycomb structure, with a 1-mm diameter central hole (accommodating the central, high-intensity part of the X-ray pulse), and the rest of the holes having approximately same size. The length of the structure would be $\sim 2 \mathrm{~cm}$. As the halo part of the beam has a relatively small power density, this "plug" would not be damaged.

The other option would be introducing a Penning discharge into one of the cells, to ionize the Xe ions and direct them by the magnetic field away from the line-of sight (Fig. 4). Of course, in the steady state, the discharge will not absorb the atoms. They will leave it after surface recombination. What is important, however, is that they will leave the discharge isotropically scattered. This will reduce the particle flux to the main vacuum chamber at least by two orders of magnitude compared to the value determined by Eq. (24).

Other options to further reduce the gas load from the attenuator include commercially available differiential pumping sections that could be added at the expense of overall length. Needless to say, the whole system would become much simpler and shorter if the aperture diameter could be reduced from $1 \mathrm{~cm}$ to, say, $0.5 \mathrm{~cm}$.

\section{Summary and discussion}

We have discussed two versions of the attenuation cell. Both do not rely on the use of any moving parts and should therefore be very reliable in operation. Requirements to the vacuum system are modest for the 1-mm aperture attenuator and somewhat more demanding for the 1-cm aperture attenuator. The absorbing gas circulates in essentially a close loop. A remarkable advantage of the gaseous attenuators is the simplicity of control over the attenuation: a mere change of the gas pressure allows one to do that. The total length of a small-aperture cell is $1 \mathrm{~m}$, and the total length of the large-aperture cell is $4 \mathrm{~m}$. In the latter case, the gas load on the accelerator vacuum LOS is probably too large, and some additional elements would have to be installed to reduce it. If the aperture can be reduced from $1 \mathrm{~cm}$ to $0.5 \mathrm{~cm}$, all parameters of the system improve, and there would be no need in additional elements.

We have not tried to optimize either attenuator system (in particular, by varying such parameters as the gas pressure in the central cell, and the lengths of the cells). On the other hand, this memo provides sufficient information for such an optimization to be 
done in the future. Generally, the system looks very robust, with a significant potential for improvements.

At a very high attenuations $\sim 10^{4}$, the attenuation becomes very sensitive to the column density $N\left(\mathrm{~cm}^{-2}\right)$. From Eqs. (6) and (7), it is clear that

$$
\frac{\Delta A}{A}=\frac{\Delta N}{N} \ln A
$$

For $A \sim 10^{4}$, to maintain the attenuation to $\pm 1 \%$, one has to maintain the relative variation of column density to $\pm 0.1 \%$. If one monitored and regulated the gas pressure in the central cell, the most probable source of the variation in the column density would be the variation in temperature. This problem can be eliminated if one uses the gauges that are sensitive to the gas density and not to the gas pressure. Note that this effect is present also when one uses solid-state attenuators (like Be filters), because the column density changes when the temperature changes (due to the volumetric expansion).

At high attenuation, some non-negligible contribution to the total value of $A$ will come from part of the X-ray trajectory outside the main attenuation cell; especially close to the nozzle where the density is high. It will be necessary therefore to carry out a direct calibration of the system and the sensitivity of the attenuator's column density to the vacuum LOS pressure needs to be quantified. For the calibration purposes, the column density could be measured by: 1) measuring the energy losses of an electron beam with the energy of a few $\mathrm{MeV}$ injected through the entrance aperture, 2) measuring the attenuation of an X-ray source placed on axis, or 3) measuring the column density with a laser. The calibration also needs to quantify possible attenuation fluctuations which may be caused by turbulence in the initial part of the nozzle where the density is still high. We believe such fluctuations will be small because the pressure and the Reynolds number of the flow is low, however this must be experimentally verified. As there are no moving parts in the system, one can expect a high stability of the attenuation (including the statistical pattern of fluctuations if they are present) provided the gas pressure in the main attenuation cell is kept constant. Therefore, the majority of the calibration can be carried out off-line.

There is a possiblility for condensation of Xe at the exit from the nozzle 3 in Fig. 2, where the gas temperature drops well below evaporation temperature of a liquid Xe $\left(-108^{0} \mathrm{C}\right)$. We have not examined the significance of this effect for the proposed attenuators (if condensation does occur it could even be beneficial). Nonetheless, data from experiments with supersonic nozzles [5] indicate that condensation becomes 
important only at much higher initial pressures; in the range of 10 atmospheres and above.

\section{Acknowledgment}

This work was performed under the auspices of the U.S. Department of Energy by the University of California Lawrence Livermore National Laboratory under contract No. W-7405-Eng-48, and by Stanford University, Stanford Linear Accelerator Center under contract number DE-AC03-76SF00515.

\section{References}

1. I.K. Kikoin (Ed.). "Tables of Physical Quantities" (Moscow, Atomizdat, 1976), (in Russian).

2. B.L. Henke, E.M. Gullikson, J.C. Davis. Atomic Data and Nuclear Data Tables, 54, 181 (1993).

3. L.D. Landau, E.M. Lifshitz. Fluid Mechanics (Oxford, Pergamon Press), 1987.

4. D.M. Hoffman, B. Singh, J. H. Thomas, III (Eds.). "Handbook of Vacuum Science and Technology" (Academic Press, 1998), p. 183.

5. T. Ditmire et al. Nature, 386, 54 (1997).

\section{Figure captions}

Fig. 1 Spatial structure of the X-ray beam. We neglect the beam angular divergence.

Fig. 2 Layout of the $10^{4}$ attenuator for the central part of the beam. 1-central cell of a high gas pressure; 2- gas inlet; 3-curved nozzle directed to the accelerator; 4analogous nozzle directed to the experimental chamber; 5-entrance orifice of the nozzle (1 mm diameter); 6-exit orifice; 7-deflector of a supersonic jet; 8intermediate cell; 9-nozzle with a special diaphragm designed to provide a large divergence of the beam entering the final chamber 10; 11-exit hole (1 mm diameter); 12-a tube with baffles.

Fig. 3 Layout of the 100-fold attenuator for the halo region. 1-central cell of a high gas pressure; 2-entrance orifice of a tube 3 with inner baffles; 4-first intermediate cell; 5-nozzle with a special diaphragm designed to provide a large divergence of the beam entering the chamber $6 ; 7$ baffled tube; 8 -final cell. 
Fig. 4 Penning discharge cell that can be placed in cell 8 (Fig. 3). C - two cathodes; Acylindrical anode made of a transparent mesh with a hole matching the size of the X-ray beam; B-the magnetic field oriented along the axis of the discharge (compact coils are not shown). 


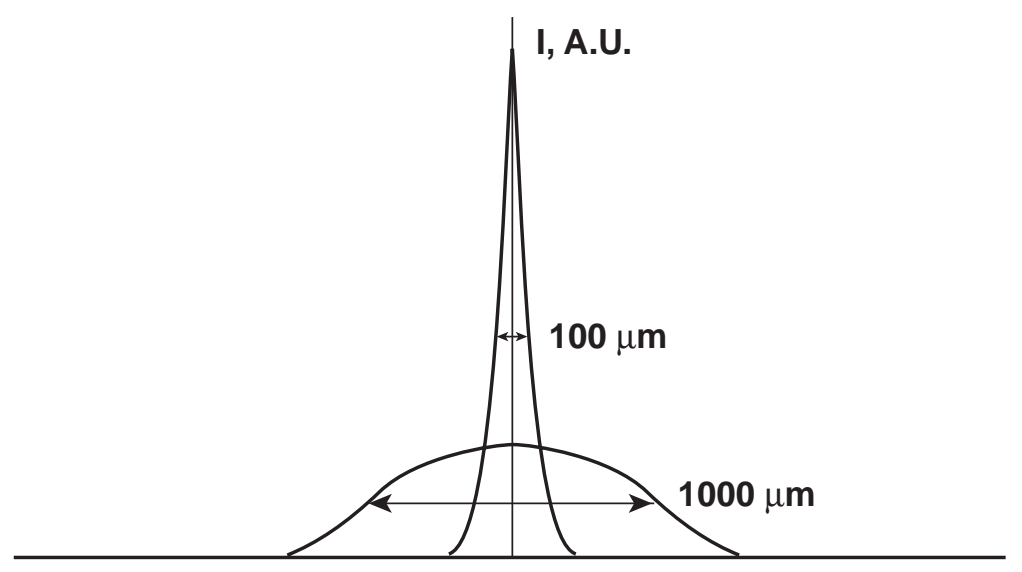

Figure 1

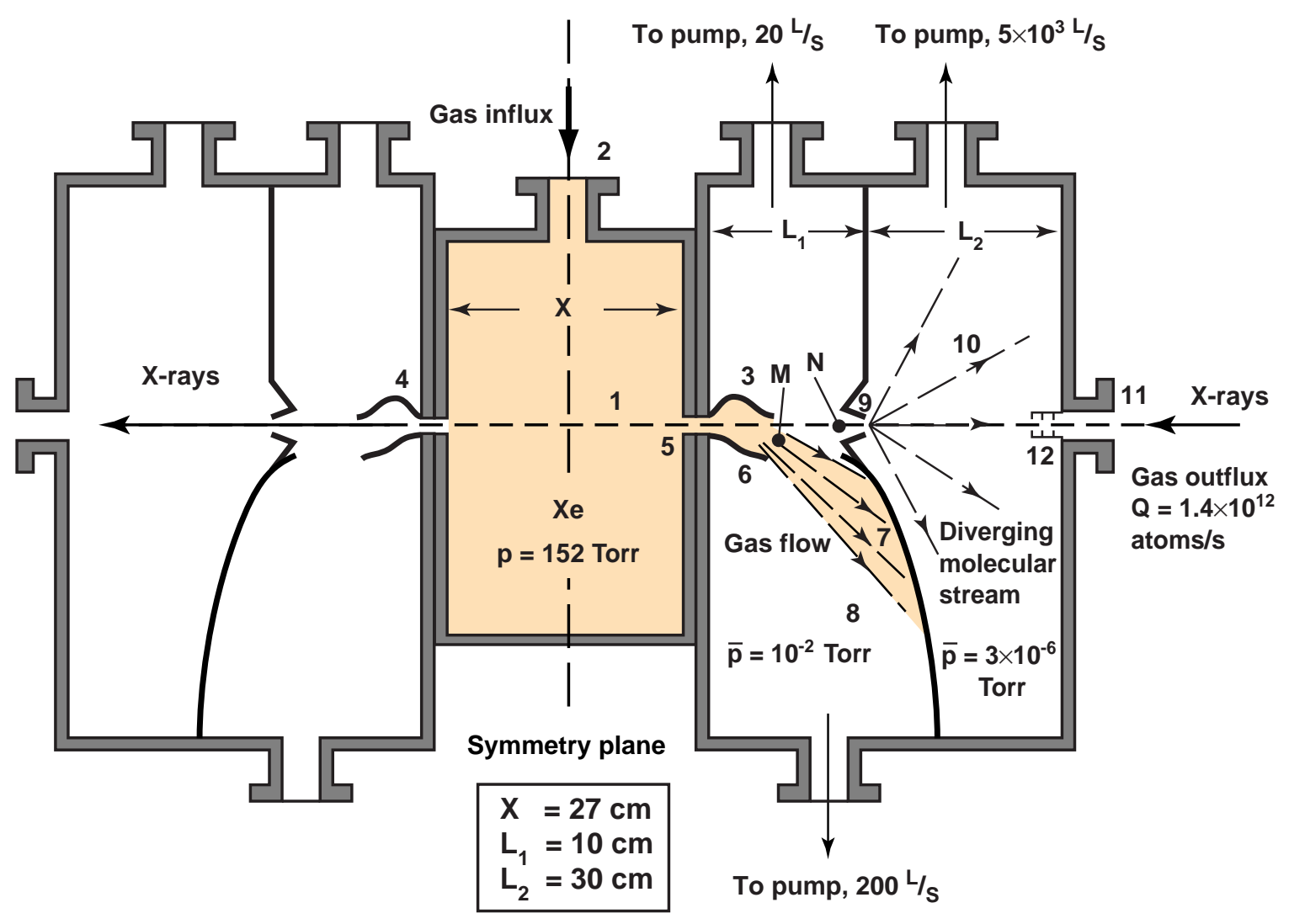

Figure 2 


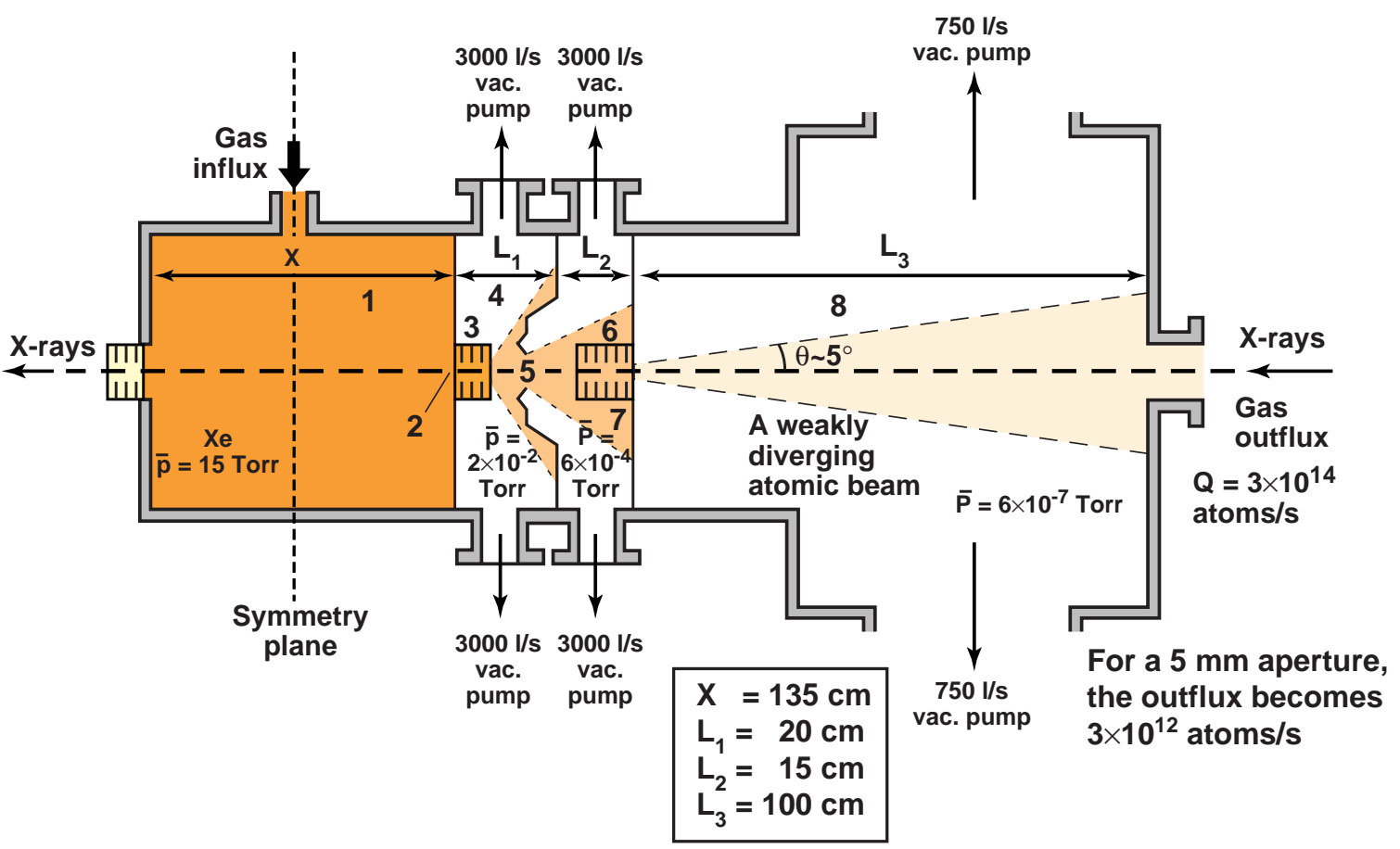

Figure 3

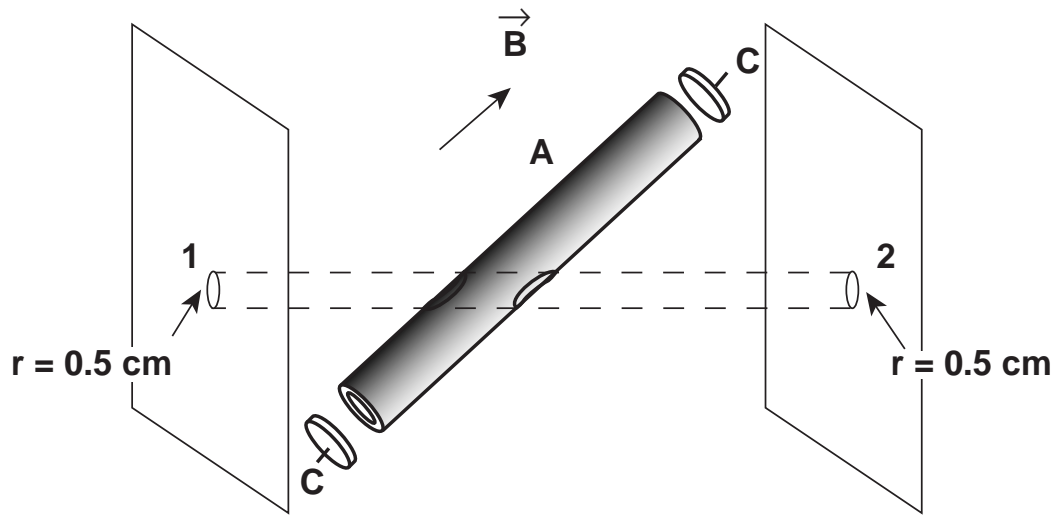

Figure 4 